\title{
Research in Reform of Information Technology Application in Preschool
}

\author{
Zhenfang Liu" ${ }^{1,}$ a, Dan Suen ${ }^{2, b}$ \\ ${ }^{1}$ Jinhua Polytechnic, Jinhua, China \\ ${ }^{2}$ Zhejiang Normal University, Jinhua, China \\ áliuzf3075@163.com, bcone.sun@yahoo.com
}

\begin{abstract}
Keywords: Preschool Education, the Orientation of Growth, Information Technology, Application Research

Abstract. Preschool education is an important part of basic education, the problem of preschool education informationization should be taken into account by educators. This article aims to investigate and research whether learning or growth should be the orientation of preschool education, thus to explore the problem of preschool education based on children's growth and information technology applications based on the orientation of children's growth.
\end{abstract}

\section{Dilemma facing preschool Education in China: Orientation of Learning or Growth}

Game-playing is the basic orientation in traditional preschool education, which is similar to growth orientation and later transformed into learning orientation with the wave of examination-oriented education, and now preschool education in China is learning-oriented. However, findings of recent preschool research support the orientation of growth. Chinese preschool education requires a revolutionary change to follow up with the pattern of growth of child and to wisely choose the orientation of growth and development for children [1].

It is worrying that the amount of learning-oriented kindergarten can be said to be overwhelming in China. This kind of preschool education will inevitably pursue so-called good grades at the cost of growth and living of children, which obviously has the order reversed. Is it worthwhile for preschool education to get the good results but abandon the healthy growth of preschool children? Therefore, growth orientation should be replaced by learning orientation. The growth orientation takes children's daily life as a way to conduct education, and takes the growth of children as its main educational purpose and task. Although preschool learning still exists in preschool education and even a lot of learning contents are similar with learning orientation, there are major differences in their status and proportion. The growth orientation of preschool education is the root way to promote the growth and cultivation of human, which pays much attention to the inside growth of children, namely physical and mental development of children. So there is a significant divergence between learning orientation and growth orientation in learning objectives, educational content, educational methods and educational evaluation.

\section{Growth Orientation: The Direction of Preschool Education Reform in China}

Game-playing is the Fundamental Form of Kindergarten Curriculum. Main purpose of preschool education is to provide a healthy growth for children, game is the basic form and main content of kindergarten curriculum. It is children's nature to play and playing is their original form of life. The so-called generative teaching as its essence is to respect and consciously use the original lives of children, in which children themselves are unaware, free and addicted into self-exploration activities. In fact, it is precisely what teachers should do to transform children's desire of self-activity to educational mission, not allowing unrestrained freedom. So it is bound to be a higher requirement for preschool education.

Kindergarten Curriculum should be integrated into Daily Life among Children. Life is an education, education is life itself. Kindergarten curriculum should be integrated in children's daily life. Consciously respect the lives of children in the natural state is a premise of the original ecology of 
children's lives. When concentrating the original ecology of children's life, teachers should not forget the importance of the original ecology of life for the growth of children. Adults should save enough blank space for children in their educational background, and consciously integrate the knowledge and ability into children' lives, so that children can have sufficient time and independent space. Nature leaves the freedom for children to enjoy its undisturbed 'gift' which are even not visible for both adults and children, but it is the nature of children to enjoy them physically and instinctively. In the children's opinion, the game and unfettered life is as important as the sun and rain for life. There is still requirements for learning in children's original ecological life, as demonstrated by the children natural state, to be curious, to explore, to ask questions and even to take risks. When education becomes an autonomous needs of children, then the dream of education is life itself will become a reality. 'Negative Educational theory' by Rousseau and "Education is Life itself" by Dewey are worthy of careful consideration [2].

On the Basis of Direct Experience to get Direct Experience for Children. Children's language skills and basic life skills are coming from direct experience in real life, so learning should be experience-based for children. Physical development of children does not just requires food, but also cultural conditions. Due to the limitations of the level of children's culture and practical experience, cultural factors are mainly from radio, television, posters and other information media, so it is passive for them to draws attainment attitude. In the meanwhile, for children, living means growth, so preschool life orientation and growth orientation are consistent. Compared with activities expressing their vitality, learning is secondary. Main purpose of preschool education should be allowing children to live naturally, study calmly, in order to obtain direct experience and grow up happily and healthily. If preschool education reform is conducted on the basis of learning orientations, utilizing information technology to provide children with traditional difficult knowledge, morality and skill, it is only a different way to push children to learn by changing the traditional arbitrary way into those flexible, intelligent method. It is only a reform in quantity when carrying out reform on learning orientation, and there will be no substantial change. Preschool education in China demands a conceptual and fundamental revolution, to achieve the fundamental change from orientation of learning to growth.

\section{Reform of Information Technology Application in Preschool Education}

IT application in preschool has its own peculiarities. Because the age among children in preschool is relatively young (typically 3-7 years old), IT application in preschool education should be reflected in improving kindergarten teachers' information literacy and strengthening the construction of information environment, and all these things should be based on the growth orientation.

Improving the Information Literacy of Kindergarten Teacher. The investigation of eastern coastal kindergarten presidents and teachers' IT ability shows that: (1) the number of full-time IT teacher is too small in kindergarten; (2) information skill of kindergarten teachers is unsatisfactory; (3) application of information technology in classroom needs to be strengthened; (4) informatization awareness in management level needs to be enhanced [3].

Thus, it is believed that: first, IT training for kindergarten presidents and teachers should be conducted as early as possible; second, encourage kindergarten teachers to integrate information technology into teaching curriculum and activities, encourage government to construct a professional platform to provide preschool teachers with various resources for preschool teachers to exchange opinions and thoughts, download leaning materials and etc., which is very important for improving kindergarten teachers' ability to use information technology, and also beneficial for the professional development of kindergarten teachers [4].

Strengthening the Construction of Information Environment in Preschool. Survey finds that the kindergarten has been attaching great importance to the construction of IT hardware environment, there are certain amount of kindergartens (especially first class kindergarten, demonstration kindergarten) equipped with basic computers (access to the Internet), but with a little computer classroom. Although the hardware configuration and network building in preschool have built a good 
material foundation for the application of information technology, the laggard software configuration and lacking of educational software are major obstacles to the smooth development of information technology in kindergarten. Investigation shows that the majority of the principals are keeping active attitudes towards the importance of information technology applications in the kindergarten, but in practice there are still some misunderstandings in educational philosophy. Information technology has brought new opportunities and challenges for educational reform and development in kindergarten, which requires kindergarten managers and teachers to change their ideas and actively adapt to the new situation.

Integrating Information Technology into Professional Development of Teacher Teaching and Growth of Children. Teacher's capability of using information technology is the core component of the information literacy in information society. Contemporary kindergarten teachers should have the ability of 'using computer and network technology to obtain information related to preschool education, creatively developing and enriching educational resources, flexibly using a variety of information to solve practical problems in kindergarten.' At the same time, it should be recognized that information literacy of preschool teachers is also a booster for the professional development of kindergarten teachers.

First, awareness of enhancing ability of integrating information technology into curriculum for preschool teachers should be improved. Integration of information technology and curriculum is more than simply regarding IT as a facilitating presentation tool for teacher teaching, not using information technology for the purpose of information technology usage. As professor He Kekang mentioned, the real integration of information technology and curriculum in teaching is to implement a new teaching structure through the application of information technology, such as games and learning reflecting the growth orientation of young children.

Second, kindergarten should actively carry out theory and practice of information technology in education, to form a suitable IT education application mode for their own schools. The survey shows that there are a little researches related to information technology in kindergarten, kindergarten teachers' scientific consciousness is insufficient. In traditional education, there are no special requirements for preschool teachers to conduct basic research. In this information era, preschool teacher is not only practitioners of modern education, and also a discoverer of the modern education regulation. Therefore, teachers' capabilities to conduct research should be a new requirement for all teachers in the new century.

Third, the current situation reveals that there is a considerable number of kindergartens which cannot afford to open information technology courses, which should be gradually improved by equipped these kindergartens with full-time kindergarten teachers in information technology, creation of information technology courses, weekly schedule some time to let children operate the computer; Kindergarten which doesn't have the condition for carrying out information technology curriculum should cooperate with local kindergarten, to utilize their computers to conduct various forms of information technology education. Study has proved that children in 4-5 years old who takes computer courses, including recognizing the difference between left and right, up and down, computing, reading, storytelling, music, graphics and color, figure out the shapes of letters, keyboard training and free colorful drawings and etc., could cultivate their ability of innovation, thus get fully prepared for their further primary school computer lesson. Research also proves that conducting curriculum in Kindergarten with multimedia computer can promote the comprehensive development of the overall quality of children. In the meanwhile, the television is also an important medium to improve children's information literacy. Work together with home-school to make more effort for the growth orientation of children.

Fourth, kindergarten teachers' ability to apply information technology should be included in the assessment of the promotion requirements. Combining flexible educational concept with rigid policy constrains to achieve a win-win preschool education informationation in utilizing information technology to accomplish the common growth for both children and teachers. 


\section{Conclusion}

Applications of information technology based on the preschool development orientation has brought about an unprecedented high-quality requirements for the kindergarten teachers. With the increasingly rich and complex of preschool educational content, life-oriented, game-oriented and modernization of preschool education courses, gradual integration of curriculum goals and flexible and diverse teaching methods, emergence of modern educational technology and the professional development of preschool teachers, these all require an improvement in information literacy for preschool teachers.

\section{Acknowledgements}

Article is founded by Zhejiang Province Philosophy and Social Planning Project 'Investigation of Preschool Education Resources Allocation in Zhejiang Province' (project number: 12JCJY03YBM).

\section{References}

[1] Xiaodong Liu, Preschool needs a Revolutionary Change in China, Journal of Educational Development. (2005)26-28. In Chinese.

[2] Xiaodong Liu, Knowledge and Growth: What is More Important in Preschool Education, Journal of Educational Development. (2005)28-30. In Chinese.

[3] ZhenFang Liu, Investigation and Analysis of Information Literacy among Preschool Teachers, Modern Educational Technology. (2010)64-48. In Chinese.

[4] ZhenFang Liu, Thinking to Enhance Application of Information Literacy among Preschool Teachers, Teachers Exposition. (2014)2-3. In Chinese. 